\title{
Ejection Dynamics of a Simple Liquid from Individual Carbon Nanotube Nozzles
}

\author{
Manuel Melle-Franco ${ }^{a, b}$ and Francesco Zerbetto ${ }^{b}$ \\ Istituto Nazionale di Scienza e Tecnologia dei Materiali, Italy \\ Dipartimento di Chimica “G. Ciamician”, Università di Bologna, V. F. Selmi 2, 40126, \\ Bologna, Italy
}

AUTHORS EMAIL ADDRESS (manuel.mellefranco@unibo.it; francesco.zerbetto@unibo.it)

\section{Computational background}

To describe the carbon structure, we employed the Brenner potential.[1] The CarbonArgon interactions were described by the relevant part of the MM3 potential,[2] which reads

$$
V(r)=\varepsilon\left[184000 \exp \left(-12\left(\frac{r}{r_{v}}\right)-2.25\left(\frac{r}{r_{v}}\right)^{6}\right)\right]
$$

where $r$ is the interatomic distance, $r_{v}$ is the sum of the van der Waals radii and $\varepsilon$ is the well depth that is calculated as the arithmetic average of the values of the two atoms; $\mathrm{r}(\mathrm{Ar})=1.990 \AA, \mathrm{r}(\mathrm{C})=1.960 \AA, \varepsilon(\mathrm{Ar})=0.268 \mathrm{kcal} \mathrm{mol}^{-1}, \varepsilon(\mathrm{C})=0.056 \mathrm{kcal} \mathrm{mol}^{-1}$. All the calculations were performed with a modified version of the TINKER molecular mechanics/dynamics software package.[3-5] 
[1] Brenner, D.W. Phys. Rev. B, 1990, 42, 9458-9471.

[2] Allinger, N.L.; Yuh, Y.H.; Lii, J.-H. J. Am. Chem. Soc. 1989, 23, 8551-8566.

[3] Ponder, J. W.; Richards, F. J. J. Comput. Chem. 1987, 8, 1016.

[4] Kundrot, C. J.; Ponder, W.; Richards, F. J. J. Comput. Chem. 1991, 12, 402.

[5] Dudek, M. J.; Ponder, J. W. J. Comput. Chem. 1995, 16, 791. 


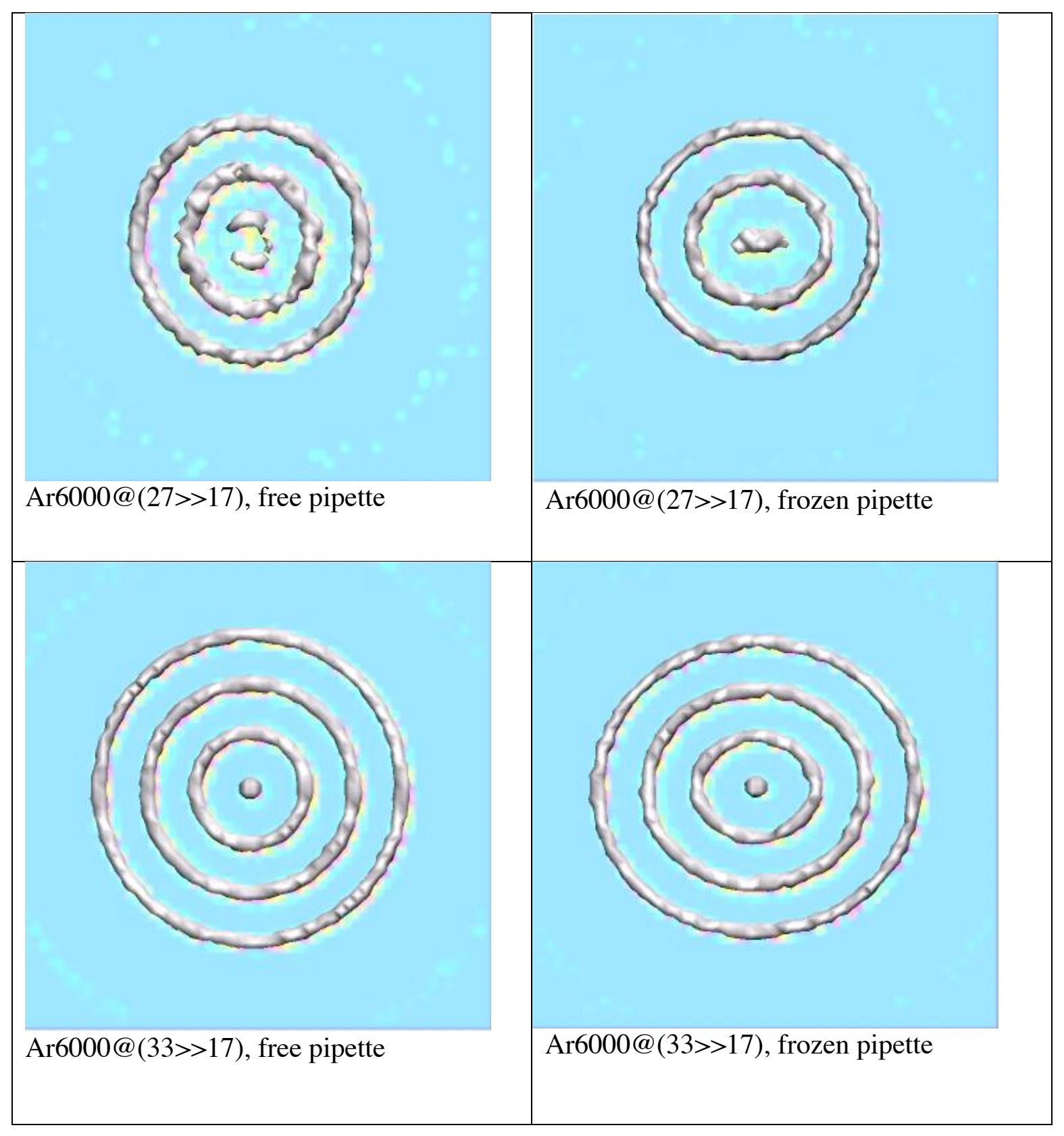




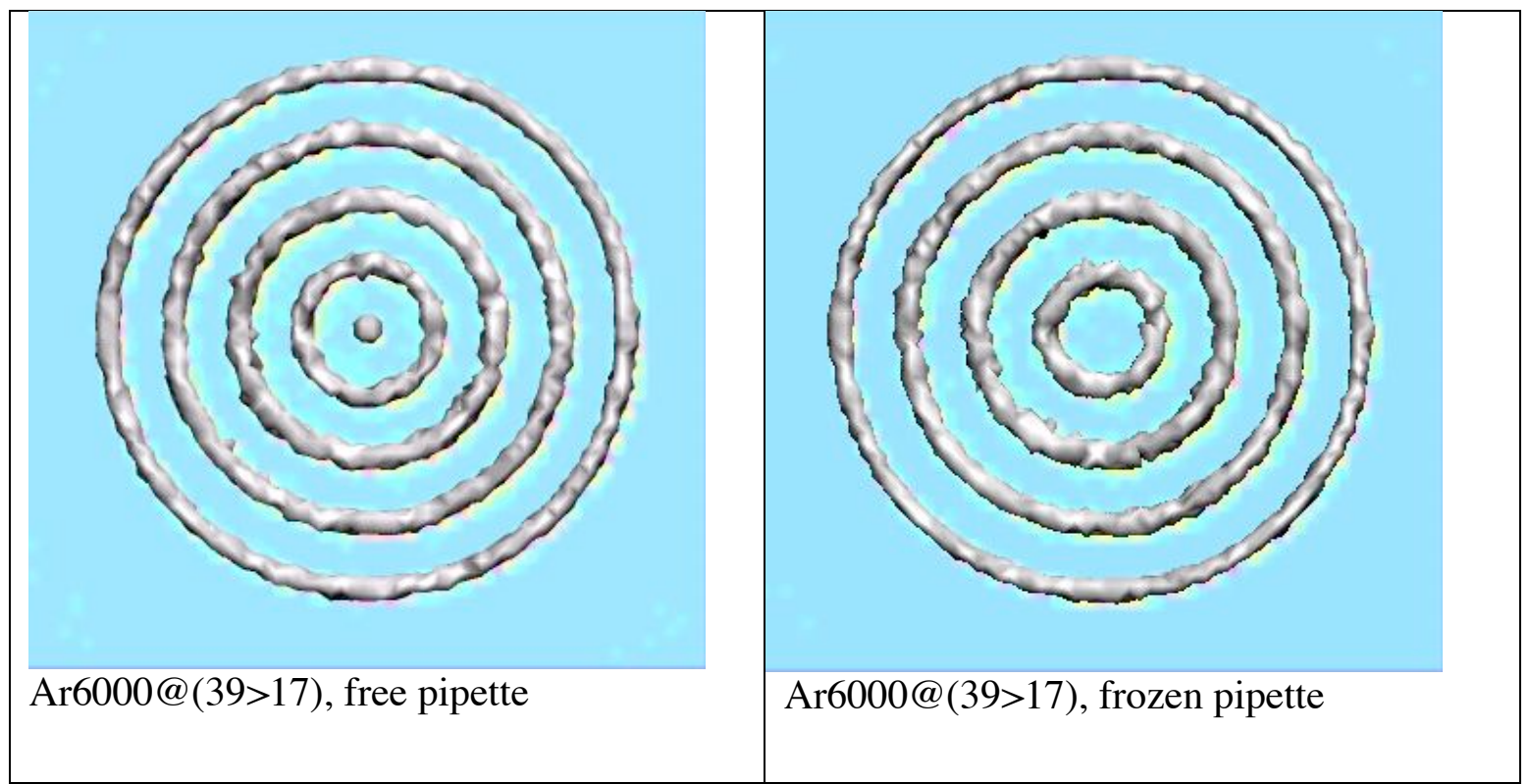

Figure SI 1. Comparison of the inlet flow density inside different nanonozzles. Left the carbon structure of the pipette is frozen, right the pipette is allowed to vibrate freely. 